\title{
Terminal-Dependent Statistical Inference for the Integral Form of FBSDE
}

\author{
Qi Zhang ${ }^{1,2}$ \\ ${ }^{1}$ School of Mathematics, Shandong University, Jinan 250100, China \\ ${ }^{2}$ College of Mathematics, Qingdao University, Qingdao 266071, China \\ Correspondence should be addressed to Qi Zhang; qizhang8409@gmail.com
}

Received 14 June 2013; Revised 4 September 2013; Accepted 22 September 2013

Academic Editor: Vasile Dragan

Copyright ( 2013 Qi Zhang. This is an open access article distributed under the Creative Commons Attribution License, which permits unrestricted use, distribution, and reproduction in any medium, provided the original work is properly cited.

\begin{abstract}
Backward Stochastic Differential Equation (BSDE) has been well studied and widely applied. The main difference from the Original Stochastic Differential Equation (OSDE) is that the BSDE is designed to depend on a terminal condition, which is a key factor in some financial and ecological circumstances. However, to the best of knowledge, the terminal-dependent statistical inference for such a model has not been explored in the existing literature. This paper is concerned with the statistical inference for the integral form of Forward-Backward Stochastic Differential Equation (FBSDE). The reason why I use its integral form rather than the differential form is that the newly proposed inference procedure inherits the terminal-dependent characteristic. In this paper the FBSDE is first rewritten as a regression version, and then a semiparametric estimation procedure is proposed. Because of the integral form, the newly proposed regression version is more complex than the classical one, and thus the inference methods are somewhat different from those designed for the OSDE. Even so, the statistical properties of the new method are similar to the classical ones. Simulations are conducted to demonstrate finite sample behaviors of the proposed estimators.
\end{abstract}

\section{Introduction}

The Backward Stochastic Differential Equation (BSDE) was first presented by Bismut [1] for the linear case and by Pardoux and Peng [2] for the general case. The solution of a BSDE consists of a pair of adapted processes $\left(Y_{t}, Z_{t}\right)$ satisfying

$$
-d Y_{t}=g\left(t, Y_{t}, Z_{t}\right) d t-Z_{t} d B_{t} ; \quad Y_{T}=\xi,
$$

where $g$ is the generator, $B_{t}$ is the standard Brownian motion, and $\xi$ is the terminal condition. Usually the terminal condition is designed as a random variable with given distribution. If $g$ meets certain conditions, the BSDE has a unique solution. The integral form of the BSDE can be expressed as

$$
Y_{t}=\xi+\int_{t}^{T} g\left(s, Y_{s}, Z_{s}\right) d s-\int_{t}^{T} Z_{s} d B_{s}
$$

The study history of the BSDE was relatively short but progressed rapidly. In addition to the interesting mathematical nature, its extensive applications gained more and more attentions; see for example Peng [3], Pardoux and
Peng [4], Pardoux and Tang [5], Peng and Wu [6], Ma and Yong [7], and Nualart and Schoutens [8]. Duffie and Epstein [9] used the BSDE to describe the consumer preferences under uncertain economic environment (i.e., the stochastic differential utility). El Karoui and Quenez [10] stated that in financial markets, prices of many important derivative securities could be solved by a certain BSDE. Lin et al. [11] used an extended statistical model to describe an ecological problem. Furthermore, the BSDE is closely related to nonlinear partial differential equation, more generally, the inseparability of nonlinear semigroup or stochastic control problems. Meanwhile, this type of equation appears frequently in mathematical finance as pointed out by Quenez [12]. Recently, Delong [13] introduced the most recent advances in BSDE (including FBSDE) and applied BSDE with jumps to insurance and finance fields.

In terms of the backward equation, within a complete market it serves to characterize the dynamic value of replicating portfolio $Y_{t}$ with a final wealth $\xi$ and a special quantity $Z_{t}$ that depends on the hedging portfolio. Especially when the randomness of $(g, \xi)$ of BSDE comes from the state of 
the forward equation, the corresponding equation is proved to be a Forward-Backward Stochastic Differential Equation (FBSDE), which can be expressed as

$$
d Y_{t}=-g\left(X_{t}, Y_{t}, Z_{t}, t\right) d t+Z_{t} d B_{t} ; \quad Y_{T}=\xi,
$$

with $X_{t}$ satisfying

$$
d X_{s}=\mu\left(s, X_{s}\right) d s+\sigma\left(s, X_{s}\right) d B_{s} .
$$

Compared to the Ordinary Stochastic Differential Equation (OSDE) that contains an initial condition, the solution of the FBSDE is affected by the terminal condition $Y_{T}=\xi\left(X_{T}\right)$. As is well known, there exist a number of parametric and nonparametric methods to deal with estimation and test for the OSDE. However, these methods can not be directly employed to infer the BSDE and FBSDE because the two models are related to a terminal condition.

For the FBSDE defined above, the statistical inference was investigated initially by Su and Lin [14], Chen and Lin [15], and a relevant model which was proposed by Lin et al. [11]. However, they did not take the terminal condition into account in the inference procedure. In the framework of the FBSDE mentioned above, the terminal condition is additional, which is not nested into the equation. Thus, there is an essential difficulty to use the terminal condition to refine the inference procedure. As a result, their methods fail to cover the full problems given in the FBSDE.

As well the FBSDE could turn to the integral form:

$$
\begin{gathered}
Y_{t}=\xi+\int_{t}^{T} g\left(s, X_{s}, Y_{s}, Z_{s}\right) d s-\int_{t}^{T} Z_{s} d B_{s}, \\
d X_{t}=\mu\left(t, X_{t}\right) d t+\sigma\left(t, X_{t}\right) d B_{t} ; \quad X_{0}=x .
\end{gathered}
$$

In this paper I focus only on the integral form because it contains the terminal condition as an additive term of the equation. With such a construction, a terminal-dependent inference could be built. I am concerned with the semiparametric estimation of the FBSDE in this paper. Note that $Z_{t}$ is usually unobservable and $g$ can not be completely specified in the financial market. The problems of interest are therefore to give both proper estimations of the generator $g$ and the process $Z_{t}$ based on observed data $\left(X_{t}, Y_{t}\right)$ and the terminal condition $\xi$. As an initial investigation, this paper only considers the model with generator being parametric structure; that is to say, $g$ can be written in the form of $g=$ $g\left(\theta, t, Y_{t}, Z_{t}\right)$, where $\theta$ is an unknown parameter vector. Even so, such a simplified form is widely used in financial markets, and, furthermore, the proposed methods can be extended to the other complicated forms.

It is worth mentioning that the key point of the method is the use of the integral equation rather than the differential equation. This change leads to a completely new work among the existing researches. Unlike the forward equation, because of the integral, the cumulative error appears not neglectable; nevertheless, the resultant estimation is still asymptotically unbiased for the condition of mixing dependency of $X_{t}$ attached. Another difference from the ordinary model is that the generator contains the unobservable process $Z_{t}$, and then it is necessary to estimate $Z_{t}$ first. After plugging the estimator of $Z_{t}$ into the generator, I could infer generator $g$ with the newly proposed methods.

The paper is organized as follows. In Section 2, the FBSDE is first rewritten as a special regression, and, by this representation, the estimation procedure for the FBSDE with linear generator is designed. Next I discuss the asymptotic properties in Section 3. A supplement for the inference of equation is suggested, and an extension for nonlinear model is briefly discussed in Section 4. Simulation study is proposed in Section 5 to illustrate the methods. The proofs of the theorems are presented in Section 6.

\section{Terminal-Dependent Semiparametric Estimation for the FBSDE}

2.1. Model and Its Statistical Version. I consider the integral form of the standard FBSDE:

$$
\begin{gathered}
Y_{t}=\xi+\int_{t}^{T} g\left(s, X_{s}, Y_{s}, Z_{s}\right) d s-\int_{t}^{T} Z_{s} d B_{s} \\
d X_{t}=\mu\left(t, X_{t}\right) d t+\sigma\left(t, X_{t}\right) d B_{t} ; \quad X_{0}=x,
\end{gathered}
$$

where $\left\{B_{t}\right\}_{t \geq 0}$ is the Brownian motion and $\xi$ is a smooth function. Here the generator $g$ is a function of $t, Y_{t}$, and $Z_{t}$, with $Z_{t}$ being usually unobservable. Furthermore, the adapted process $Y_{t}, Z_{t}$ and terminal condition could be indicated as a function of $X_{t}$. As is known to everyone, the existence and uniqueness result of the FBSDE have been studied elaborately. This section is intended to represent the FBSDE as a statistical framework and then address the proper estimators of $g$ and $Z_{t}$ based on observed data $\left\{X_{t}, Y_{t}\right\}$ and the terminal condition $\xi$.

To recast the model (6) as a statistical model, I first examine the property of the last term of the first equation in (6). By the property of Itô integral and the relation between the two equations in (6), I have

$$
E\left(\int_{t}^{T} Z_{s} d B_{s}\right)=0, \quad \operatorname{Var}\left(\int_{t}^{T} Z_{s} d B_{s}\right)=E\left(\int_{t}^{T} Z_{s}^{2} d s\right) .
$$

Then I regard $-\int_{t}^{T} Z_{s} d B_{s}$ as error and consequently rewrite the first equation of model (6) as

$$
Y_{t}=\xi+\int_{t}^{T} g\left(s, Y_{s}, Z_{s}\right) d s+\epsilon_{t}
$$

where $\epsilon_{\mathrm{t}}$ is the error term with mean zero and bounded variance, and the adapted process $Y_{t}, Z_{t}$ and terminal condition $\xi$ depend on $X_{t}$ via the second equation of (6).

Remark 1. It seems that formula (8) proposes a regression that is determined by both expectation and variance frameworks. However, such a regression is quite unlike the classical one. In the newly defined structure, although the expectation of the error is zero, the conditional expectation of the error is nonzero. Even so, the resultant estimation is asymptotically unbiased, and thus the consistency of the estimators 
defined below still holds because of the condition of mixing dependency of $X_{t}$ given below; for details see the following theorems and the proofs of the theorems.

Given the initial calendar time point $t_{1}$, I record the observed time series data $\left\{X\left(t_{i}\right), Y\left(t_{i}\right), i=1, \ldots, n\right\}$ at the equally spaced time points $\left\{t_{i}=t_{1}+(i-1) \Delta, i=1, \ldots, n\right\} \subseteq$ $[0, T]$. Denote $\Delta_{i}=t_{i+1}-t_{i}(=\Delta)$ for $1 \leq i \leq n-1$ and $\Delta_{n}=T-t_{n}$. Note that $\Delta_{n}$ is the distance between the last observation time $t_{n}$ and the terminal time $T$; indeed it may be quite large and then makes the following formula (9) inaccurate. Therefore I first assume $\Delta_{n}$ small enough, that is, $\Delta_{n}=O(\Delta)$, and then propose an adjustment in Section 4 for the case with larger $\Delta_{n}$. On the other hand, since the distribution of $\xi$ is supposed known, I can get the samples $\left\{\xi_{i}, 1 \leq i \leq k\right\}$ for $k \geq 1 / \Delta$.

In this section I assume $g$ can be expressed as linear function $g\left(t, Y_{t}, Z_{t}\right)=a+b Y_{t}+c Z_{t}$, where $a, b$, and $c$ are unknown parameters. Then the model (8) can be approximately rewritten as

$$
\begin{gathered}
Y\left(t_{i}\right)=\widehat{\xi}+\sum_{j=i}^{n}\left(a+b Y\left(t_{j}\right)+c Z\left(t_{j}\right)\right) \Delta_{j}+O(\Delta)+\epsilon_{i}+v_{s}, \\
E\left(\epsilon_{i}\right)=0, \quad \operatorname{Var}\left(\epsilon_{i}\right)=\sum_{j=i}^{n} \Delta_{j} Z^{2}\left(t_{j}\right)+O(\Delta), \\
i=1, \ldots, n,
\end{gathered}
$$

where $\widehat{\xi}=(1 / k) \sum_{i=1}^{k} \xi_{i}$ and $\nu_{s}=\xi-\widehat{\xi}$, satisfying $E\left(\nu_{s}\right)=0$ and $\operatorname{Var}\left(\nu_{s}\right)=\operatorname{Var}(\xi)(1+(1 / k))$.

This is the statistical version of (8), a new regression model. It is worth mentioning that the new model (9) is somewhat different from the classical regression; that is, in addition to the mean-variance structure, the new one has a complicated structure and contains a terminal information.

2.2. Semi-Parametric Estimation for the FBSDE. I now turn to estimating unknown parameter vector $\beta=(a, b, c)^{\tau}$ in model (9). While the generator contains unobservable interesting process $Z_{t}$, it is necessary to estimate $Z$ for plugging the estimator into the generator firstly. After that, the common parametric estimation methods can be employed to estimate parameters.

Concerning inference of $Z_{t}$, despite the connection between $Z_{t}$ and the variance of $\epsilon_{t}$ in (9), the second formula of (9) is related to the weighted sum of $Z_{t}^{2}$, which causes inconvenience for estimating $Z_{t}$ by residual-based method. I now adopt a difference-based method instead.

To this end, consider the FBSDE model (6), motivated by Stanton [16], for the Markov process $\left\{X_{s}\right\}_{t \leq s \leq T}$ which follows the SDE,

$$
d X_{s}=\mu\left(s, X_{s}\right) d s+\sigma\left(s, X_{s}\right) d B_{s}
$$

the infinitesimal generator $\mathscr{L} f\left(t, X_{t}\right)$ is defined as

$$
\begin{aligned}
\mathscr{L} f\left(t, X_{t}\right) \\
=\lim _{\Delta \rightarrow 0^{+}} \frac{1}{\Delta}\left\{E\left[f\left(t+\Delta, X_{t+\Delta}\right) \mid X_{t}=x\right]-f(t, x)\right\} \\
=\frac{\partial f}{\partial t}+\mu(t, x) \frac{\partial f(t, x)}{\partial x}+\frac{1}{2} \sigma^{2}(t, x) \frac{\partial^{2} f(t, x)}{\partial x^{2}},
\end{aligned}
$$

where the bivariate function $f(\cdot, \cdot)$ satisfies the sufficient smoothing condition [17]. By Taylor's expansion, the condition expectation $E\left[f\left(t+\Delta, X_{t+\Delta}\right) \mid X_{t}=x\right]$ can be expressed as

$$
\begin{aligned}
E[ & \left.f\left(t+\Delta, X_{t+\Delta}\right) \mid X_{t}=x\right] \\
= & f(t, x)+\mathscr{L} f(t, x) \Delta+\frac{1}{2} \mathscr{L}^{2} f(t, x) \Delta^{2} \\
& +\cdots+\frac{1}{n !} \mathscr{L}^{n} f(t, x) \Delta^{n}+O\left(\Delta^{n+1}\right),
\end{aligned}
$$

which implies that when the time increment $\Delta \rightarrow 0$, the firstorder approximation formula for $\mathscr{L} f(t, x)$ can be given by

$$
\begin{aligned}
& \mathscr{L} f(t, x) \\
& =\frac{1}{\Delta} E\left[f\left(t+\Delta, X_{t+\Delta}\right)-f\left(t, X_{t}\right) \mid X_{t}=x\right]+O(\Delta) .
\end{aligned}
$$

In addition, we need the following generalized Feynman-Kac formula. Let $v$ be a $\mathscr{C}^{1,2}$ function, and suppose there exists a constant $C$ such that, for each $(t, x)$,

$$
|v(t, x)|+\left|\partial_{x} v(t, x) \sigma(t, x)\right| \leq C(1+|x|),
$$

and $v$ is the solution of the following system of quasilinear parabolic partial differential equation

$$
\begin{gathered}
\mathscr{L} v(t, x)+f\left(t, x, v(t, x), \partial_{x} v(t, x) \sigma(t, x)\right)=0, \\
v(T, x)=\phi(x) .
\end{gathered}
$$

Then $Y_{s}^{t, x}=v\left(s, X_{s}^{t, x}\right), Z_{s}^{t, x}=\partial_{x} v\left(s, X_{s}^{t, x}\right) \sigma\left(s, X_{s}^{t, x}\right)$, a.s., where $\left(Y_{s}^{t, x}, Z_{s}^{t, x}\right)$ is the unique solution of the FBSDE, based on the result in Pardoux and Peng [4].

Denote $\left(Y_{s}^{t, x}, Z_{s}^{t, x}\right)$ by $\left(Y_{s}, Z_{s}\right)$, respectively, for short. By using the Taylor's expansion of $f(s, x)=\left(v(s, x)-v\left(t, X_{t}\right)\right)^{2}$,

$$
\begin{aligned}
\mathscr{L} f(s, x)= & 2\left(v(s, x)-v\left(t, X_{t}\right)\right) \mathscr{L} v(s, x) \\
& +v_{x}^{2}(s, x) \sigma^{2}(s, x) ;
\end{aligned}
$$

then $\mathscr{L} f\left(t, X_{t}\right)=v_{x}^{2}\left(t, X_{t}\right) \sigma^{2}\left(t, X_{t}\right)$, and

$$
\begin{aligned}
& \mathscr{L} f\left(t, X_{t}\right) \\
& =\frac{1}{\Delta} E\left[\left(v\left(t+\Delta, X_{t+\Delta}\right)-v\left(t, X_{t}\right)\right)^{2} \mid X_{t}=x\right]+O(\Delta) .
\end{aligned}
$$


Finally an approximation of $Z_{t}^{2}$ could be expressed as

$$
\begin{aligned}
Z_{t}^{2} & =v_{x}^{2}\left(t, X_{t}\right) \sigma^{2}\left(t, X_{t}\right)=\mathscr{L} f\left(t, X_{t}\right) \\
& =\frac{1}{\Delta} E\left[\left(v\left(t+\Delta, X_{t+\Delta}\right)-v\left(t, X_{t}\right)\right)^{2} \mid X_{t}=x\right]+O(\Delta)
\end{aligned}
$$

that is,

$$
Z_{t}^{2}=\frac{1}{\Delta} E\left[\left(Y_{t+\Delta}-Y_{t}\right)^{2} \mid X_{t}=x\right]+O(\Delta)
$$

By (19), I regard $Z^{2}\left(x_{0}\right)$ as point-wise nonparametric regression function. For simplicity, here the N-W kernel estimator is taken as an example of nonparametric smooth estimators:

$$
\widehat{Z}^{2}\left(x_{0}\right)=\frac{\sum_{i=1}^{n-1} \Delta^{-1}\left(Y\left(t_{i+1}\right)-Y\left(t_{i}\right)\right)^{2} K_{h}\left(X\left(t_{i}\right)-x_{0}\right)}{\sum_{i=1}^{n-1} K_{h}\left(X\left(t_{i}\right)-x_{0}\right)},
$$

where $K_{h}(\cdot)=K(\cdot / h) / h, K(\cdot)$ is the kernel function satisfying the regularity condition given below and $h$ is the bandwidth or smoothing parameter. Similarly, if $Z_{t}$ also depends on $t$ besides $X_{t}$, the corresponding estimator could be

$$
\begin{aligned}
& \widehat{Z}^{2}\left(x_{0}, t_{0}\right) \\
& =\frac{\sum_{i=1}^{n-1} \Delta^{-1}\left(Y\left(t_{i+1}\right)-Y\left(t_{i}\right)\right)^{2} K_{h_{X}}\left(X\left(t_{i}\right)-x_{0}\right) K_{h_{t}}\left(t_{i}-t_{0}\right)}{\sum_{i=1}^{n-1} K_{h_{X}}\left(X\left(t_{i}\right)-x_{0}\right) K_{h_{t}}\left(t_{i}-t_{0}\right)} .
\end{aligned}
$$

Since having calculated $\widehat{Z}_{t}^{2}$, I plug it in the first formula of (9), obtaining

$$
\begin{aligned}
& Y\left(t_{i}\right) \approx \widehat{\xi} \\
&+\sum_{j=i}^{n}\left(a+b Y\left(t_{j}\right)+c \widehat{Z}\left(t_{j}\right)\right) \Delta_{j} \\
&+O\left(\Delta_{i}\right)+\epsilon_{i}+v_{s}, \quad i=1, \ldots, n .
\end{aligned}
$$

From the above, it is simple to deduce the estimator of $\beta=(a, b, c)^{\tau}$ with common parametric methods, the least square method for example, by minimizing

$$
\sum_{i=1}^{n}\left(Y\left(t_{i}\right)-\widehat{\xi}-\sum_{j=i}^{n}\left(a+b Y\left(t_{j}\right)+c \widehat{Z}\left(t_{j}\right)\right) \Delta t_{j}\right)^{2} .
$$

For simplicity, denote

$$
V=\frac{1}{T}\left(\begin{array}{c}
Y\left(t_{1}\right)-\widehat{\xi} \\
\cdots \\
Y\left(t_{n}\right)-\widehat{\xi}
\end{array}\right) \text {, }
$$

$$
\begin{gathered}
U=\frac{1}{T}\left(\begin{array}{ccc}
\sum_{j=1}^{n} \Delta_{j} & \sum_{j=1}^{n} Y\left(t_{j}\right) \Delta_{j} & \sum_{j=1}^{n} Z\left(t_{j}\right) \Delta_{j} \\
\ldots & \ldots & \ldots \\
\Delta_{n} & Y\left(t_{n}\right) \Delta_{n} & Z\left(t_{n}\right) \Delta_{n}
\end{array}\right), \\
\widehat{U}=\frac{1}{T}\left(\begin{array}{ccc}
\sum_{j=1}^{n} \Delta_{j} & \sum_{j=1}^{n} Y\left(t_{j}\right) \Delta_{j} & \sum_{j=1}^{n} \widehat{Z}\left(t_{j}\right) \Delta_{j} \\
\cdots & \ldots & \ldots \\
\Delta_{n} & Y\left(t_{n}\right) \Delta_{n} & \widehat{Z}\left(t_{n}\right) \Delta_{n}
\end{array}\right) .
\end{gathered}
$$

Finally, I can write the estimator as

$$
\widehat{\beta}=\left(\widehat{U}^{\tau} \widehat{U}\right)^{-1} \widehat{U}^{\tau} V
$$

\section{Asymptotic Results}

The following two theorems are concerned with asymptotic properties of the estimators deduced in the previous section.

First of all, I lead in several conditions.

(a) $X_{1}, \ldots, X_{n}$ are $\rho$-mixing dependent; namely, the $\rho$ mixing coefficients $\rho(l)$ satisfy $\rho(l) \rightarrow 0$ as $l \rightarrow \infty$, where

$\rho(l)=\sup _{E\left(X_{i+l} X_{i}\right)-E\left(X_{i+l}\right) E\left(X_{i}\right) \neq 0} \frac{\left|E\left(X_{i+l} X_{i}\right)-E\left(X_{i+l}\right) E\left(X_{i}\right)\right|}{\sqrt{\operatorname{Var}\left(X_{i+l}\right) \operatorname{Var}\left(X_{i}\right)}}$

with $X_{i}=X\left(t_{i}\right)$.

(b) $\left|Z_{i}\right| \leq C$ (a.s.) uniformly for $i=1, \ldots, n$, where $C$ is a positive constant and $Z_{i}=Z\left(t_{i}\right)$.

(c) The continuous kernel function $K(\cdot)$ is symmetric about 0 , with a support of interval $[-1,1]$, and

$$
\begin{gathered}
\int_{-1}^{1} K(u) d u=1, \quad \sigma_{K}^{2}=\int_{-1}^{1} u^{2} K(u) d u \neq 0 \\
\int_{-1}^{1}|u|^{j} K^{k}(u) d u<\infty \quad \text { for } j \leq k=1,2 .
\end{gathered}
$$

(d) As $n \rightarrow \infty$,

$$
\frac{1}{n} U^{\tau} U \stackrel{p}{\longrightarrow} \Sigma, \quad \frac{1}{n T^{2}} U^{\tau} U \operatorname{Var}(\epsilon) \stackrel{p}{\longrightarrow} \Omega,
$$


where the matrix $\Sigma$ is nonsingular and satisfies

$$
0<C_{1}<\lambda_{\min }(\Sigma)<\lambda_{\max }(\Sigma)<C_{2}<\infty,
$$

with $\lambda_{\min }(\Sigma)$ and $\lambda_{\max }(\Sigma)$ being the smallest and largest eigenvalues of $\Sigma$, respectively.

The condition (a) is commonly used for the weakly dependent process; see for example Rosenblatt [18, 19], Kolmogorov and Rozanov [20], Bradley and Bryc [21], Lin and $\mathrm{Lu}$ [22], and $\mathrm{Su}$ and Lin [14]. The condition (b) is also reasonable because, as is shown by (19), $Z_{t}$ can be regarded as the deviation between the adjacent two observations. The condition (c) is standard for nonparametric kernel function, and the condition (d) is obviously common because it describes the property of average. Furthermore, as remarked in the previous section, to express the estimator related to $X_{t}$ rather than model variables $Y_{t}$ and $Z_{t}$, I apply conditions mainly on the latent variable $X_{t}$, including the stationary $\rho$ mixing Markov character used in the following theorems. Actually the process $\left\{Y_{t}\right\}$ may be unstationary.

Theorem 2. Besides the conditions (a), (b), and (c), suppose that $X_{i} \in\left(x_{0}-h, x_{0}+h\right)$ is a stationary $\rho$-mixing Markov process with the $\rho$-mixing coefficients satisfying $\rho(l)=\rho^{l}$ for $0<\rho<1$ and has a common probability density $p(x)$ satisfying $p\left(x_{0}\right)>0, Z^{2}\left(x_{0}\right)>0$. Furthermore, functions $p(x)$ and $Z(x)$ have continuous two derivatives in a neighborhood of $x_{0}$. As $n \rightarrow \infty$, if $n h \rightarrow \infty, n h^{5} \rightarrow 0$ and $n h \Delta^{2} \rightarrow 0$, then

$$
\sqrt{(n-1) h}\left(\widehat{Z}^{2}\left(x_{0}\right)-Z^{2}\left(x_{0}\right)\right) \stackrel{\mathscr{D}}{\longrightarrow} N\left(0, \frac{Z^{4}\left(x_{0}\right) J_{K}}{p\left(x_{0}\right)}\right) .
$$

The proof is presented in Section 6. The asymptotic result in the theorem is standard for nonparametric kernel estimator, and here undersmoothing is used to eliminate asymptotic bias.

Theorem 3. In addition to the condition of Theorem 2, if the condition (d) holds, then as $n \rightarrow \infty$,

$$
\sqrt{n}(\widehat{\beta}-\beta) \stackrel{d}{\longrightarrow} N\left(0, \sigma^{2} \Sigma^{-1}+\Sigma^{-1} \Omega \Sigma^{-1}\right),
$$

where $\sigma^{2}=\operatorname{Var}(\xi / T)$.

The proof is also presented in Section 6. The result is eventually standard in the sense of asymptotic normality with the convergence rate of order $\sqrt{n}$. As was shown in the remark given in the previous section, even the conditional mean of error of the model is nonzero, the newly proposed estimation is consistency because of the mixing dependency; for details see the proof of Theorem 3. Furthermore, because of the terminal condition, the asymptotic variance is larger than that without use of the terminal condition.

\section{Supplement and Extension}

4.1. Supplement. As is mentioned in Section 2.1, when the last observation $t_{n}$ is far away from the terminal $T$, the new model
(9) appears inaccurate. In this case I need an adjustment to obtain a relatively accurate model. The main steps of adjustment are defined as follows: first I ignore the terminal condition to obtain both the accurate model and parameters estimations limited in $\left(0, t_{n}\right)$; next I estimate the unobservable variables in the interval $\left(t_{n}, T\right)$ by the first step estimated model; finally, I substitute the estimators for the unobservable variables in $\left(t_{n}, T\right)$ and build a relatively accurate model defined in the whole interval $[0, T]$ and related to the terminal condition.

For arbitrary $t \leq t_{n}$

$$
Y_{t}=Y_{n}+\int_{t}^{t_{n}} g\left(s, Y_{s}, Z_{s}\right) d s-\int_{t}^{t_{n}} Z_{s} d B_{s}
$$

This equation is accurate and thus I can get the estimators $\widehat{g}$ and $\widehat{Z}_{t}$ of $g$ and $Z_{t}$ for $t \leq t_{n}$ by the methods given in Section 2. When $t>t_{n}$, this method is however unsuitable for estimating $Z_{t}$ because it cannot be extrapolated to the interval $\left(t_{n}, T\right)$, so I attempt to complete the data within this interval.

Set $t_{n}<t_{n+1}<\cdots<t_{n+l}<T$. Discretize model (32) and write its forward linear version as

$$
\begin{aligned}
Y\left(t_{i+1}\right)= & -\left(\left(b-\frac{1}{\Delta_{i}}\right) Y\left(t_{i}\right)+c Z\left(t_{i}\right)\right) \Delta_{i} \\
& +Z\left(t_{i}\right)\left(B_{i+1}-B_{i}\right) .
\end{aligned}
$$

Similar to formula (9), the expectation-variance structure is shown as

$$
\begin{aligned}
& E\left(Y\left(t_{i+1}\right) \mid Y\left(t_{i}\right), Z\left(t_{i}\right)\right) \\
& \quad=-\left(\left(b-\frac{1}{\Delta_{i}}\right) Y\left(t_{i}\right)+c Z\left(t_{i}\right)\right) \Delta_{i}, \\
& \operatorname{Var}\left(Y\left(t_{i+1}\right) \mid Y\left(t_{i}\right), Z\left(t_{i}\right)\right)=\Delta_{i} Z^{2}\left(t_{i}\right) .
\end{aligned}
$$

To estimate the unobservable data $\left(Y\left(t_{i}\right), Z\left(t_{i}\right)\right)$ for $i=n+$ $1, \ldots, n+l$, I treat $Z_{t}$ as being parameterizable. It is known by, for example, Morris [23] that variance can be expressed as the quadratic function of mean for several common distributions, such as normal, gamma, binomial, negative binomial, and Poisson. For the mean-variance structure in (34), I might as well suppose the following parametric structure:

$$
\begin{aligned}
\Delta_{i} Z^{2}\left(t_{i}\right)= & \gamma_{1}+\gamma_{2}\left(\left(b-\frac{1}{\Delta_{i}}\right) Y\left(t_{i}\right)+c Z\left(t_{i}\right)\right) \Delta_{i} \\
& +\gamma_{3}\left(\left(b-\frac{1}{\Delta_{i}}\right) Y\left(t_{i}\right)+c Z\left(t_{i}\right)\right)^{2} \Delta_{i}^{2},
\end{aligned}
$$

for some parameters $\gamma_{k}$. By simply transforming and neglecting $\mathrm{O}\left(\Delta^{2}\right)$ terms, I see

$$
Z\left(t_{i}\right)=\omega_{1}+\omega_{2} \sqrt{\omega_{3}+\omega_{4} Y\left(t_{i}\right)}
$$

where $\omega_{2}=1$ or -1 , and denote $\omega=\left(\omega_{1}, \omega_{2}, \omega_{3}, \omega_{4}\right)$.

Let $\bar{Z}\left(t_{i}\right)=\omega_{1}+\omega_{2} \sqrt{\omega_{3}+\omega_{4} Y\left(t_{i}\right)}(1 \leq i \leq n)$ and plug $\bar{Z}\left(t_{i}\right)$ into (32). I then could get the estimators through 
the methods in Section 2; denote by $\widetilde{b}_{S}, \widetilde{c}_{S}$, and $\widetilde{\omega}$ the estimators of $b, c$ and $\omega$, respectively. Finally I could refine the original orbit and estimate $Z\left(t_{n+1}\right)$ one by one, more precisely,

$$
\begin{gathered}
\widetilde{Y}\left(t_{n+1}\right)=-\left(\left(\widetilde{b}_{S}-\frac{1}{\Delta_{i}}\right) Y\left(t_{n}\right)+\widetilde{c}_{S} \widetilde{Z}\left(t_{n}\right)\right) \Delta_{n}, \\
\widetilde{Z}\left(t_{n+1}\right)=\widetilde{\omega}_{1}+\widetilde{\omega}_{2} \sqrt{\widetilde{\omega}_{3}+\widetilde{\gamma}_{4} \widetilde{Y}\left(t_{n+1}\right)} .
\end{gathered}
$$

Iterating the above procedures, I obtain the complete data in $[0, T]$. Consequently, the same approaches as in Section 2 could be performed again, and a refined estimator of $g$ could be constructed.

4.2. Extension. Consider that the semiparametric models in Section 2 are of linear structure in the sense that $g$ is linearly related to parameters $a, b$, and $c$. However, some generators are nonlinear in parameters; thus the resulting model (9) will be nonlinear. For example Constantinides [24] presented the resulting model with the specification form:

$$
d X_{t}=\left(\alpha_{0}+\alpha_{1} X_{t}+\alpha_{2} \sqrt{X_{t}-\alpha_{3}}\right) d t+\sigma_{0}\left(X_{t}-\alpha_{3}\right) d B_{t}
$$

See for other examples Fan [25], Fan and Zhang [26], Chan et al. [27], and Aït-Sahalia [28].

Then, for the flexibility of modeling the above case, a nonlinear semiparametric model can be defined as

$$
\begin{gathered}
Y_{t}=\xi+\int_{t}^{T} g\left(\theta, s, Y_{s}, Z_{s}\right) d s+\epsilon_{t}, \\
\operatorname{Var}\left(\epsilon_{t}\right)=E\left(\int_{t}^{T} Z_{s}^{2} d s\right),
\end{gathered}
$$

where $\epsilon_{t}=\int_{t}^{T} Z_{s} d B_{s}$ satisfying $E\left(\epsilon_{t}\right)=0, g$ is a given function, and $\theta$ is an unknown $p$-dimensional parameter vector.

Before estimating nonlinear model (39), $Z_{t}$ can be estimated similarly by (19) or (20) because its estimator is free of the structure of $g$. Furthermore, the resulting estimator has the same asymptotic properties as in Theorem 2. Thus I only focus on the estimation of parameter vector $\theta$ here.

After plugging the estimator $\widehat{Z}_{t}$ of $Z_{t}$ into the first formula of (39), I can adopt a common method to obtain an estimator of $\theta$, for example, by minimizing

$$
\widehat{Q}(\theta)=\sum_{i=1}^{n}\left(Y_{i}-\widehat{\xi}-\sum_{j=i}^{n} \widehat{g}_{j}(\theta) \Delta t_{j}\right)^{2},
$$

where $\widehat{g}_{j}(\theta)=g\left(\theta, t_{j}, Y_{j}, \widehat{Z}_{j}\right)$. Under regularity conditions, I can also get $\hat{\theta}$ by solving the following equation:

$$
\begin{aligned}
\widehat{L}(\theta) & =\widehat{Q}^{\prime}(\theta) \\
& =-2 \sum_{i=1}^{n}\left[\left(Y_{i}-\widehat{\xi}-\sum_{j=i}^{n} \widehat{g}_{j}(\theta) \Delta t_{j}\right) \sum_{j=i}^{n} \hat{g}_{j}^{\prime}(\theta) \Delta t_{j}\right]=0,
\end{aligned}
$$

where $\widehat{Q}^{\prime}(\theta)$ denotes the derivative of $\widehat{Q}(\theta)$. By the similar arguments used in the previous section, the resultant estimator is normally distributed; the details are omitted here.

\section{Simulations}

In this section I investigate the finite-sample behaviors by simulation. Despite Theorems 2 and 3 based on stationarity of $X_{t}$, I also extend this method to nonstationary process such as Geometric Brownian Motion. I use the mean, standard deviation (STD) or mean square error (MSE) to evaluate the estimations, based on 300 repetitions. Apparently, the model with stationary condition will work better.

Example 4. Consider Cox-Ingersoll-Ross (CIR) process:

$$
d X_{t}=k\left(\theta-X_{t}\right) d t+\sigma X_{t}^{1 / 2} d B_{t} ; \quad X_{0}=x_{0} .
$$

This model describes the interest rate dynamic system and is stationary when $2 k \theta \geq \sigma^{2}$. On the other hand, the riskless asset with price per unit $P_{t}$ is conducted as follows:

$$
d P_{t}=r P_{0} d t
$$

with $r$ being the constant short rate. Let $n_{0}(t)$ and $n_{1}(t)$ denote the quantities invested in bond $P_{t}$ and asset $X_{t}$, respectively. Naturally the total wealth process $Y_{t}$ satisfies $Y_{t}=n_{0}(t) P_{t}+$ $n_{1}(t) X_{t}$. Similar with the classic self-financing FBSDE model in El Karoui et al. [29], the resulting model is

$$
\begin{gathered}
d Y_{t}=\left(n_{1} k \theta+r Y_{t}+\frac{k+r}{n_{1} \sigma^{2}} Z_{t}^{2}\right) d t+Z_{t} d B_{t} ; \quad Y_{T}=\xi\left(X_{T}\right), \\
d X_{t}=k\left(\theta-X_{t}\right) d t+\sigma X_{t}^{1 / 2} d B_{t} ; \quad X_{0}=x_{0} .
\end{gathered}
$$

Denote parameters $a=n_{1} k \theta, b=r$, and $c=(k+r) / n_{1} \sigma^{2}$. I put the equal length $\Delta=0.4$ of time period and choose sample size $n=300$. So the time interval is $[0,120]$. The terminal time is chosen as 122 , which is quite near the former one. Let $k=0.2, \theta=0.06, \sigma=0.08, r=0.05$, and $n_{0}=n_{1}=10$. In the estimation procedure, I use the Gaussian kernel defined by $K(t)=(1 / \sqrt{2 \pi}) \exp \left(-t^{2} / 2\right)$; meanwhile the optimal bandwidths would be $O\left(n^{-1 / 5}\right)$ theoretically, and popular data-driven method can also be used, such as $\mathrm{CV}$, GCV, or plug-in approach. In the simulation, I set $h=$ $\operatorname{std}(x) n^{-1 / 5}$ for simplicity. The simulation results with other choices are similar.

I present the true curves and the N-W nonparametric estimation curves for $Z_{t}$ and generator $g$ and report the mean and MSE of estimator $\hat{\beta}$ of $\beta \triangleq(a, b, c)$, respectively, in Table 1. These results show that the estimators of $a$ and $b$ work well. However, because of the plug-in estimator $\widehat{Z}_{t}$, the estimator of the coefficient $c$ has fairly large bias and the MSE. On the other hand, Figure 1 shows that the estimation curves of drift and diffusion are closed to the true ones.

Example 5. In this part I consider the case that the terminal time is far away from the last observed time, as mentioned in 


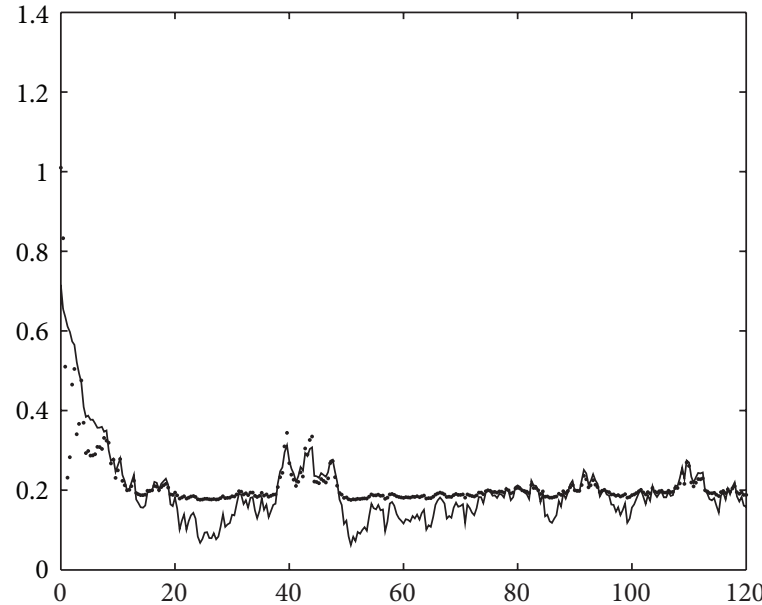

- Curve of $Z$

- Estimated curve of $Z$

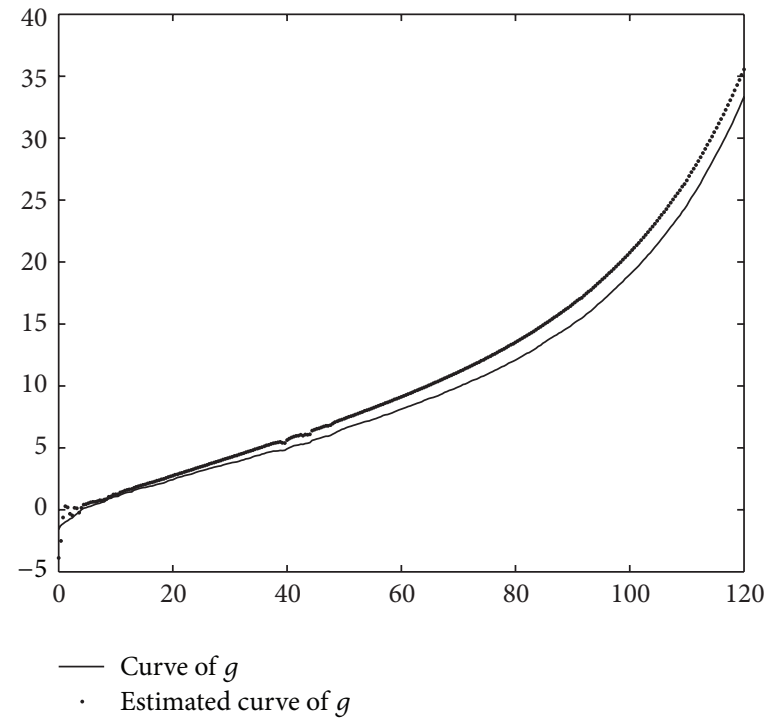

(b)

FIgURE 1: The real lines are the true curves of $Z_{t}$ and function $g$ respectively, and the dashed ones are estimated curves for them in Example 4 .

TABLE 1

\begin{tabular}{lccc}
\hline Parameter & True value & Mean & MSE \\
\hline$a$ & 0.12 & 0.1404 & 0.0089 \\
$b$ & 0.05 & 0.0503 & $1.2694 e-007$ \\
$c$ & -3.9062 & -4.5826 & 4.6295 \\
\hline
\end{tabular}

TABLE 2

\begin{tabular}{lccc}
\hline Parameter & True value & Mean & MSE \\
\hline$a$ & 0.12 & 0.1470 & 0.0319 \\
$b$ & 0.05 & 0.0490 & $1.6873 e-006$ \\
$c$ & -3.9062 & -3.8360 & 9.2929 \\
\hline
\end{tabular}

the Supplement Section. The distance between $T=124$ and $t_{n}=120$ is larger than that in Example 4 . I add 10 estimated points by the method given in Section 4 and employ the same model and parameters as before. Table 2 reports the simulation results, which tells us that the parameter estimators do not perform as well as before but still feasible. Besides, Figure 2 presents the estimated curves for $Z_{t}$ and $g$, which also perform well although they are not better than the estimations in Example 4.

Example 6. I turn to the nonstationary case in this part. Obviously when forward process $X_{t}$ does not satisfy the stationary condition, this cumulate effect induced by backward addition performs more significantly, which makes the statistical inference quite a challenge. Under this situation, I choose certain model and parameters to control the relative stationarity.
TABLE 3

\begin{tabular}{lccc}
\hline Parameter & True value & Mean & STD \\
\hline$b$ & 0.05 & 0.0473 & 0.0348 \\
$c$ & 5 & 5.7788 & 7.3388 \\
\hline
\end{tabular}

I consider a simple FBSDE as

$$
\begin{aligned}
d Y_{t} & =\left(\frac{\mu-r}{\sigma} Z_{t}+r Y_{t}\right) d t+Z_{t} d B_{t} \\
& \triangleq\left(b Y_{t}+c Z_{t}\right)+Z_{t} d B_{t} ; \quad Y_{T}=\xi\left(X_{T}\right),
\end{aligned}
$$

where $X_{t}$ is Geometric Brownian Motion for modeling stock price satisfying

$$
d X_{t}=\mu X_{t} d t+\sigma X_{t} d B_{t} ; \quad X_{0}=x,
$$

while the riskless asset is the same as formula (43), $d P_{t}=$ $r P_{0} d t$.

Firstly, let $\mu=0.1, \sigma=0.01, \Delta=0.12, n=300$, $T=36.6$, and $n_{0}=n_{1}=10$. Obviously $Z_{t}=n_{1} \sigma X_{t}$. I choose the same pattern kernel function and bandwidth $h=$ $\operatorname{std}(x) n^{-1 / 5}$. Table 3 reports the simulation results. The results show that the estimators of $b$ work well, but $c$ have larger bias and the STD because of the plug-in estimator $\widehat{Z}_{t}$. While the curves can still be fitted well, that is, the estimated curves of drift and diffusion are closed to the true ones, Figure 3 presents the estimated curves for diffusion $Z_{t}$ and drift $g$ by one simulation.

Finally, I choose $\sigma$ relatively large as 0.05 and 0.12 , which display different extension of volatilities. From Tables 4 and 5 and Figures 4 and 5, I can see that their performances are not so bad, which means that the approach could be applied more widely. 


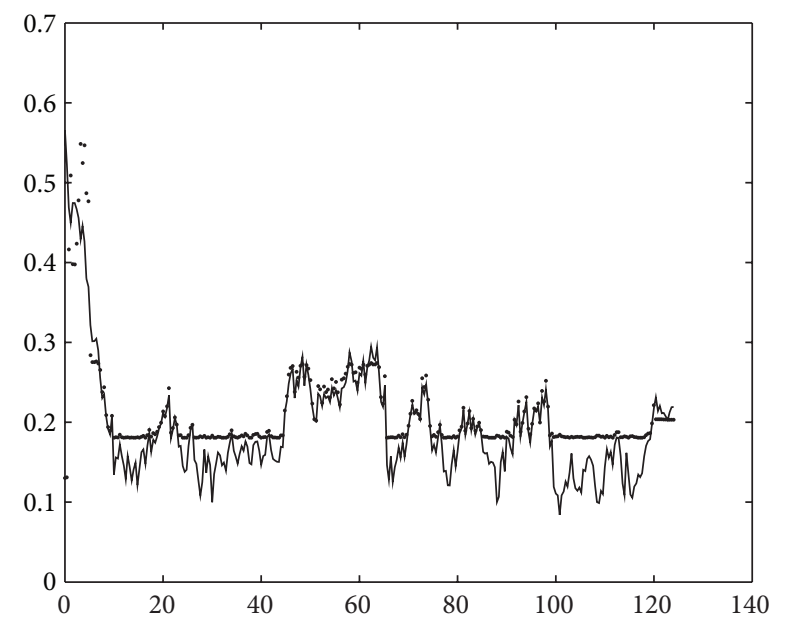

Curve of $Z$

Estimated curve of $Z$

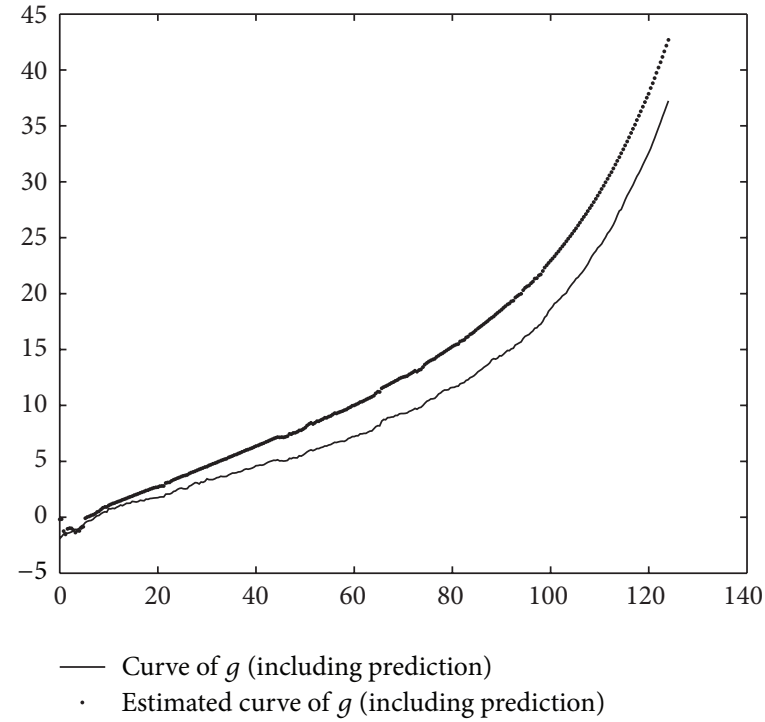

(b)

Figure 2: The real lines are the true curves of $Z_{t}$ and function $g(t)$, respectively, and the dashed ones are estimated curves for them in Example 5.

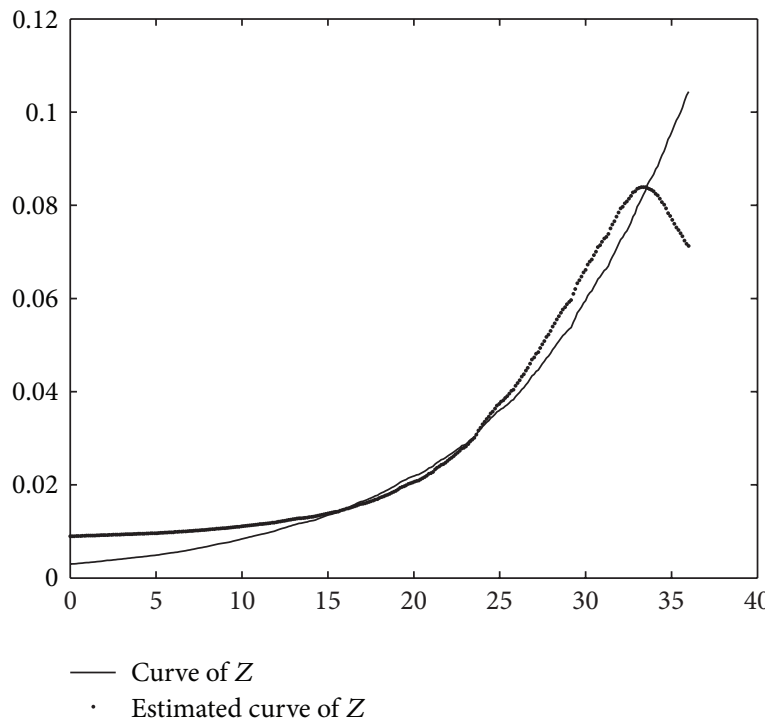

(a)

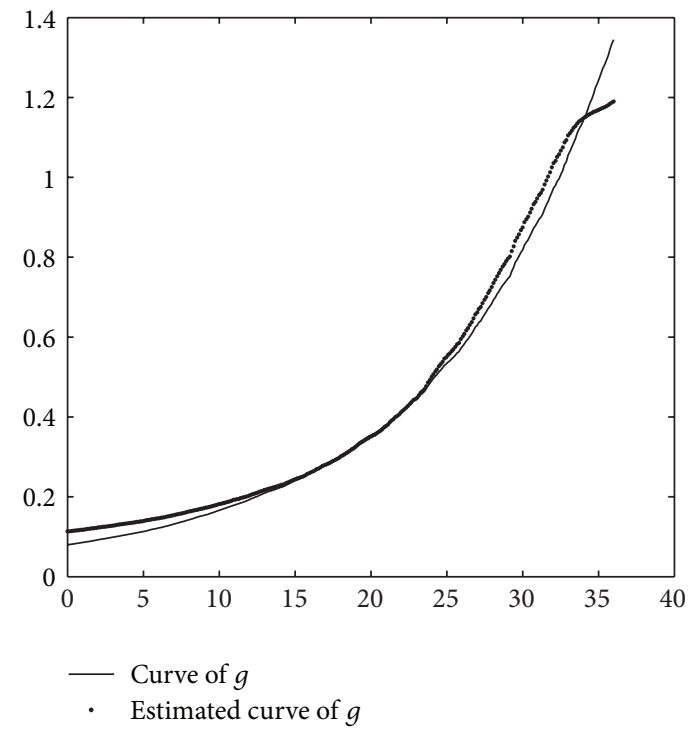

(b)

FIGURE 3: The real lines are the true curves of $Z_{t}$ and function $g(t)$, respectively, and the dashed ones are estimated curves for them in Example 6.

\section{Proofs}

Proof of Theorem 2. Denote $\mathscr{C}=\left\{X_{1}, \ldots, X_{n}, \ldots\right\}$. By the Taylor expansion and formula (19), I have

$$
\begin{aligned}
& E\left(\widehat{Z}^{2}\left(x_{0}\right) \mid \mathscr{C}\right) \\
& =\frac{\sum_{i=1}^{n-1} \Delta^{-1} K_{h}\left(X_{i}-x_{0}\right) E\left(\left(Y_{i+1}-Y_{i}\right)^{2} \mid \mathscr{C}\right)}{\sum_{i=1}^{n-1} K_{h}\left(X_{i}-x_{0}\right)}
\end{aligned}
$$

$$
\begin{aligned}
& =\frac{\sum_{i=1}^{n-1} K_{h}\left(X_{i}-x_{0}\right)\left(Z_{i}^{2}+O(\Delta)\right)}{\sum_{i=1}^{n-1} K_{h}\left(X_{i}-x_{0}\right)} \\
& =\left(\int K_{h}\left(X_{i}-x_{0}\right)\left(Z^{2}(x)+O(\Delta)\right) p(x) d x\right. \\
& \left.\quad \times\left(1+O_{p}(n h)^{-1 / 2}\right)\right)\left(\int K_{h}\left(X_{i}-x_{0}\right) p(x) d x\right. \\
& \left.\quad \times\left(1+O_{p}(n h)^{-1 / 2}\right)\right)^{-1}
\end{aligned}
$$




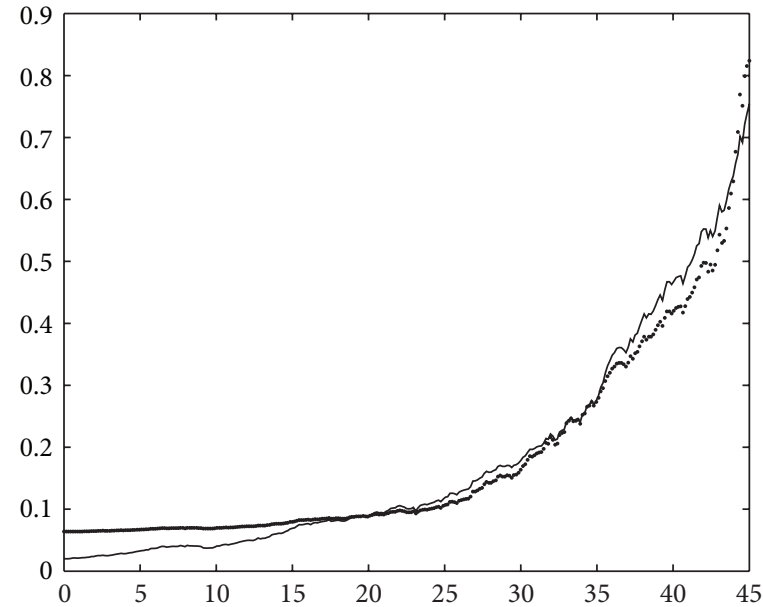

- Curve of $Z$

Estimated curve of $Z$

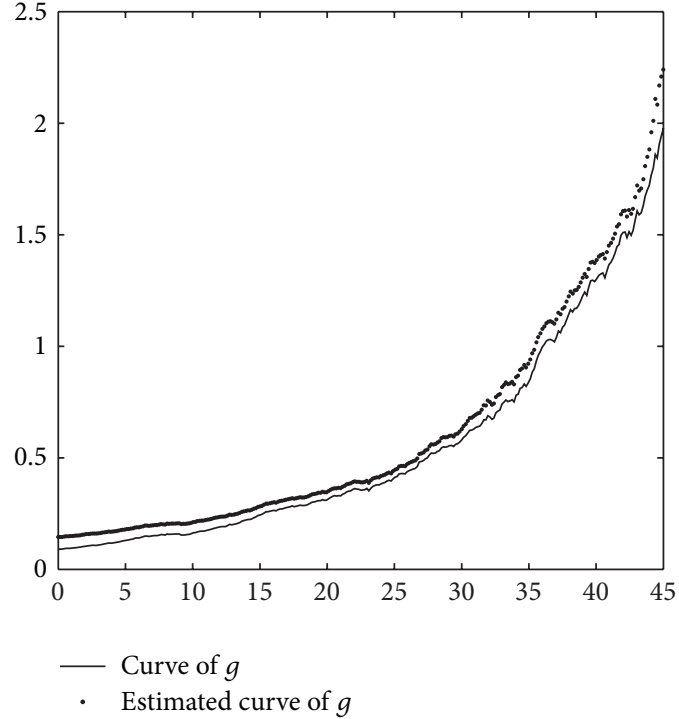

(b)

Figure 4: The real lines are the true curves of $Z_{t}$ and function $g(t)$, respectively, and the dashed ones are estimated curves for them with $\sigma=0.05$

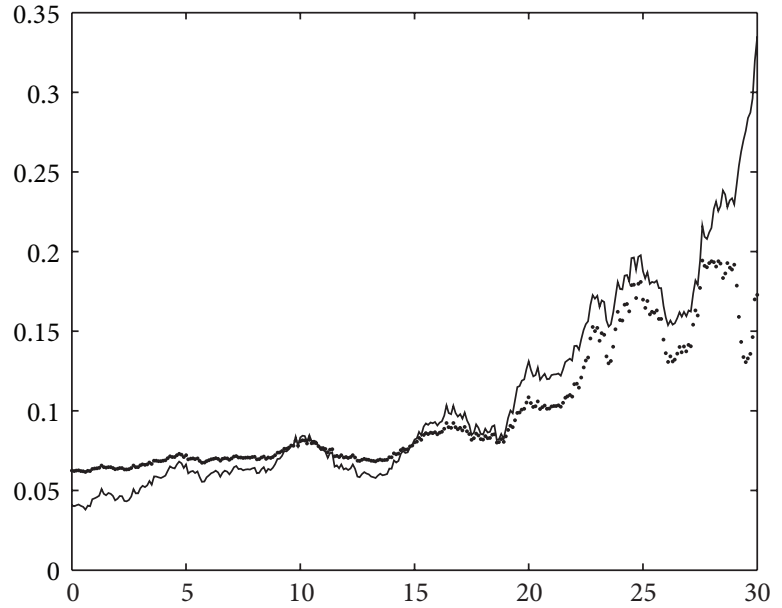

- Curve of $Z$

- $\quad$ Estimated curve of $Z$

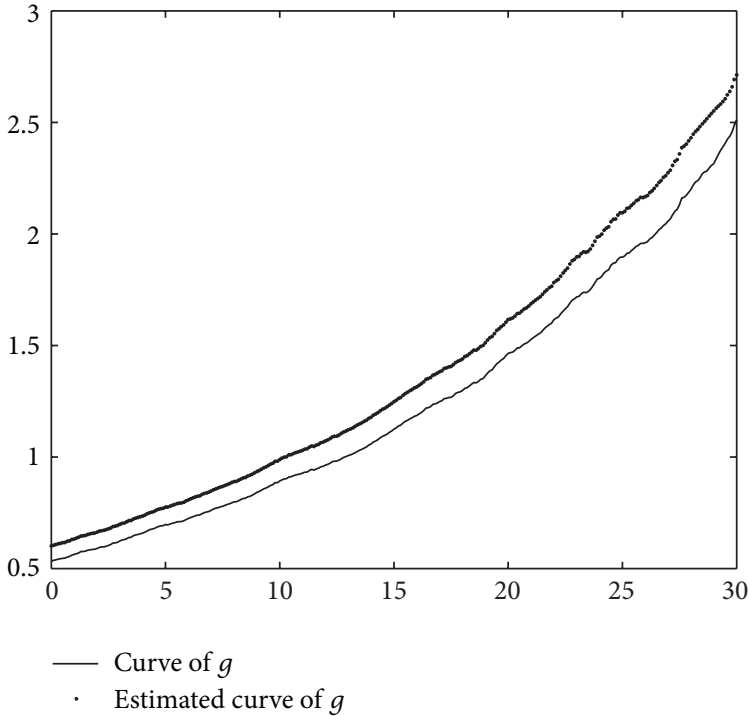

(b)

Figure 5: The real lines are the true curves of $Z_{t}$ and function $g(t)$, respectively, and the dashed ones are estimated curves for them with $\sigma=0.12$

$$
=\left(\left(Z^{2}\left(x_{0}\right)+O(\Delta)\right)\left(p\left(x_{0}\right)+\frac{1}{2} h^{2} p^{(2)}\left(x_{0}\right) \sigma_{K}^{2}+o\left(h^{2}\right)\right)=Z^{2}\left(x_{0}\right)+\frac{p^{(2)}\left(x_{0}\right)}{2 p\left(x_{0}\right)} h^{2} Z^{2}\left(x_{0}\right) \sigma_{K}^{2}+o\left(h^{2}\right)+O(\Delta)\right. \text {. }
$$$$
\left.\times\left(1+O_{p}(n h)^{-1 / 2}\right)\right)
$$$$
\times\left(\left(p\left(x_{0}\right)+\frac{1}{2} h^{2} p^{(2)}\left(x_{0}\right) \sigma_{K}^{2}+o\left(h^{2}\right)\right)\right.
$$

Furthermore,

$$
\left.\times\left(1+O_{p}(n h)^{-1 / 2}\right)\right)^{-1}
$$

$$
\begin{aligned}
& \operatorname{Var}\left(\widehat{Z}^{2}\left(x_{0}\right) \mid \mathscr{C}\right) \\
& =\frac{1}{\sum_{i=1}^{n-1} K_{h}^{2}\left(X_{i}-x_{0}\right)}
\end{aligned}
$$




$$
\begin{aligned}
\times\left\{\sum_{i=1}^{n-1} \Delta^{-2} K_{h}^{2}\left(X_{i}-x_{0}\right) \operatorname{Var}\left(\left(Y_{i+1}-Y_{i}\right)^{2} \mid \mathscr{C}\right)\right. \\
+\sum_{i=1}^{n-1} \sum_{k=1}^{n-i} \Delta^{-2} \operatorname{cov}\left(K_{h}\left(X_{i}-x_{0}\right)\left(Y_{i+1}-Y_{i}\right),\right. \\
\left.\left.K_{h}\left(X_{i+k}-x_{0}\right)\left(Y_{i+k+1}-Y_{i+k}\right) \mid \mathscr{C}\right)\right\} .
\end{aligned}
$$

From the conditions of Markov process and $\rho$-mixing coefficient,

$$
\begin{aligned}
& \mid \sum_{i=1}^{n-1} \sum_{k=1}^{n-i} \Delta^{-2} \operatorname{cov}\left(K_{h}\left(X_{i}-x_{0}\right)\left(Y_{i+1}-Y_{i}\right)\right. \\
& \left.K_{h}\left(X_{i+k}-x_{0}\right)\left(Y_{i+k+1}-Y_{i+k}\right)\right) \mid \\
& =\frac{1}{(n-1)^{2}} \\
& \quad \times \sum_{i=1}^{n-1} \sum_{k=1}^{n-i} \mid E\left((\Delta)^{-2}\left(Y_{i+1}-Y_{i}\right)^{2}\left(Y_{i+k+1}-Y_{i+k}\right)^{2}\right. \\
& \times\left(K_{h}\left(X_{i}-x_{0}\right)-E\left(K_{h}\left(X_{i}-x_{0}\right)\right)\right) \\
& \left.\quad \times\left(K_{h}\left(X_{i+k}-x_{0}\right)-E\left(K_{h}\left(X_{i+k}-x_{0}\right)\right)\right)\right) \mid \\
& =\frac{1}{(n-1)^{2}} \mid E\left(Z_{i}^{2} Z_{i+l}^{2}\left(K_{h}\left(X_{i}-x_{0}\right)-E\left(K_{h}\left(X_{i}-x_{0}\right)\right)\right)\right. \\
& \left.\times\left(K_{h}\left(X_{i+k}-x_{0}\right)-E\left(K_{h}\left(X_{i+k}-x_{0}\right)\right)\right)\right) \mid+O(\Delta) \\
& \frac{C}{(n-1)^{2} h} \sum_{i=1}^{n-1} \sum_{k=1}^{n-i} \rho^{k}=O\left(\frac{1}{n h}\right)=o(1) .
\end{aligned}
$$

Note that $\left(Y_{i+1}-Y_{i}\right) / \sqrt{\Delta}=g\left(t_{i}, Y_{i}, Z_{i}\right) \sqrt{\Delta}+Z_{i} \eta_{i}$, where $E\left(\eta_{i}\right)=0, \operatorname{Var}\left(\eta_{i}\right)=1$. Thus $\operatorname{Var}\left(\left(Y_{i+1}-Y_{i}\right) / \sqrt{\Delta}\right)=Z_{i}^{4}+O(\Delta)$ and furthermore

$$
\begin{aligned}
\operatorname{Var} & \left(\widehat{Z}^{2}\left(x_{0}\right) \mid \mathscr{C}\right) \\
= & \frac{\sum_{i=1}^{n-1} \Delta^{-2} K_{h}^{2}\left(X_{i}-x_{0}\right) \operatorname{Var}\left(\left(Y_{i+1}-Y_{i}\right)^{2} \mid \mathscr{C}\right)}{\sum_{i=1}^{n-1} K_{h}^{2}\left(X_{i}-x_{0}\right)}+O_{p}(1) \\
= & \frac{\sum_{i=1}^{n-1} K_{h}^{2}\left(X_{i}-x_{0}\right)\left(Z^{4}\left(x_{0}\right)+O(\sqrt{\Delta})\right)}{\sum_{i=1}^{n-1} K_{h}^{2}\left(X_{i}-x_{0}\right)}+O_{p}(1) \\
= & \frac{Z^{4}\left(x_{0}\right) J_{K}+O(\sqrt{\Delta})}{n h p\left(x_{0}\right)}\left(1+O_{p}(n h)^{-1 / 2}\right)
\end{aligned}
$$

To my interest, both the conditional expectation and variance are independent of $\mathscr{C}$, so the condition could be erased.
TABLE 4

\begin{tabular}{lccc}
\hline Parameter & True value & Mean & STD \\
\hline$b$ & 0.05 & 0.0580 & 0.0217 \\
$c$ & 1 & 0.9959 & 7.6034 \\
\hline
\end{tabular}

TABLE 5

\begin{tabular}{lccc}
\hline Parameter & True value & Mean & STD \\
\hline$b$ & 0.05 & 0.0557 & 0.0421 \\
$c$ & 0.4167 & 0.4227 & 6.5970 \\
\hline
\end{tabular}

From Lemma 1 of Politis and Romano [30] and the relation between the $\alpha$-mixing condition and the $\rho$-mixing condition (e.g., Theorem 1.1.1 of [22]), I can ensure that $\left\{\left(Y_{i+1}-Y_{i}\right)^{2}, i=1, \ldots, n-1\right\}$ is a $\rho$-mixing-dependent process and the mixing coefficient, denoted by $\rho_{Y}(l)$, satisfies

$$
\sum_{k=1}^{\infty} \rho_{Y}\left(2^{k}\right) \leq C \sum_{k=1}^{\infty} \rho\left(2^{k}\right)=\sum_{k=1}^{\infty} \rho^{2^{k}}<\infty,
$$

where $C$ is a positive constant. Finally, I use the Central Limit Theorems for $\rho$-mixing-dependent process (e.g., Theorem 4.0.1 of [22]) to complete this proof.

Proof of Theorem 3. I present the basic results for $(1 / \sqrt{n})(\widehat{U}-$ $U)^{\tau} U$, which leads to rate of convergence and asymptotic expansions. Similar to Cui et al. [31] or Su and Lin [14], I need the following decomposition:

$$
\begin{aligned}
\left(\frac{1}{n} U^{\tau} U\right)^{-1}-\left(\frac{1}{n} \widehat{U}^{\tau} \widehat{U}\right)^{-1} & \\
= & -\left(\frac{1}{n} U^{\tau} U\right)^{-1}\left\{\frac{1}{n}(\widehat{U}-U)^{\tau} U\right\}\left(\frac{1}{n} U^{\tau} U\right)^{-1} \\
& -\left(\frac{1}{n} U^{\tau} U\right)^{-1}\left\{\frac{1}{n} U^{\tau}(\widehat{U}-U)\right\}\left(\frac{1}{n} U^{\tau} U\right)^{-1} \\
& -\left(\frac{1}{n} U^{\tau} U\right)^{-1}\left\{\frac{1}{n}(\widehat{U}-U)^{\tau}(\widehat{U}-U)\right\}\left(\frac{1}{n} U^{\tau} U\right)^{-1} \\
& +\left\{\left(\frac{1}{n} \widehat{U}^{\tau} \widehat{U}\right)^{-1}-\left(\frac{1}{n} U^{\tau} U\right)^{-1}\right\}\left\{\frac{1}{n} \widehat{U}^{\tau} \widehat{U}-\frac{1}{n} U^{\tau} U\right\} \\
& \times\left(\frac{1}{n} U^{\tau} U\right)^{-1} \\
\triangleq & I_{1}+I_{2}+I_{3}+I_{4} .
\end{aligned}
$$

By the condition (d), $(1 / n) U^{\tau} U \stackrel{p}{\rightarrow} \Sigma$ and all eigenvalues of $\Sigma$ are bounded. Furthermore, by the uniform weak consistency kernel estimator of mixing-dependent variables, (see, e.g., $[32,33])$, I have

$$
\sup _{x}\left|\frac{1}{n-1} \sum_{i=1}^{n-1} K_{h, i}-p(x)\right|=O_{p}\left(h^{2}+\left(\frac{\log (n h)}{n h}\right)^{1 / 2}\right) .
$$


By the proof of Theorem 2, I have

$$
\begin{gathered}
E\left(\frac{1}{n}(\widehat{U}-U)^{\tau} U\right)=O\left(h^{2}\right), \\
\operatorname{Var}\left(\frac{1}{n}(\widehat{U}-U)^{\tau} U\right)=O\left(\frac{1}{\left(n^{2} h\right)^{1 / 2}}\right) .
\end{gathered}
$$

It is easy to deduce that $I_{1}=o_{p}(1 / \sqrt{n}), I_{2}=o_{p}(1 / \sqrt{n}), I_{3}=$ $o_{p}(1 / \sqrt{n})$, and $I_{4}=o_{p}(1 / \sqrt{n})$. Then I naturally get

$$
\left(\frac{1}{n} \widehat{U}^{\tau} \widehat{U}\right)^{-1}=\left(\frac{1}{n} U^{\tau} U\right)^{-1}+o_{p}\left(\frac{1}{\sqrt{n}}\right) .
$$

Denote $I_{5}=\left((1 / n) U^{\tau} U\right)^{-1}(1 / \sqrt{n})(\widehat{U}-U)^{\tau} V$. Obviously $I_{5}=$ $o_{p}(1)$. Combining the results above, I can see that

$$
\begin{aligned}
& \sqrt{n} \widehat{\beta}_{I} \\
&=\sqrt{n}\left(\widehat{U}^{\tau} \widehat{U}\right)^{-1} \widehat{U}^{\tau} V \\
&= {\left[\left(\frac{1}{n} U^{\tau} U\right)^{-1}+o_{p}\left(\frac{1}{\sqrt{n}}\right)\right] \frac{1}{\sqrt{n}} \widehat{U}^{\tau} V } \\
&=\left(\frac{1}{n} U^{\tau} U\right)^{-1} \frac{1}{\sqrt{n}} \widehat{U}^{\tau} V+o_{p}(1) \\
&=\left(\frac{1}{n} U^{\tau} U\right)^{-1} \frac{1}{\sqrt{n}} U^{\tau} V+I_{5}+o_{p}(1) \\
&=\left(\frac{1}{n} U^{\tau} U\right)^{-1} \frac{1}{\sqrt{n}} U^{\tau} V+o_{p}(1) \\
&= \sqrt{n} \beta+\left(\frac{1}{n} U^{\tau} U\right)^{-1} \frac{1}{T \sqrt{n}} \sum_{i=1}^{n} U_{i}^{\prime}\left(\epsilon_{j}+v_{s}\right)+o_{p}(1) \\
&= \sqrt{n} \beta+\left(\frac{1}{n} U^{\tau} U\right)^{-1} \frac{1}{T \sqrt{n}} \sum_{i=1}^{n} U_{i}^{\prime} \sum_{j=i}^{n} \Delta_{j}^{1 / 2} Z_{j} \varepsilon_{j} \\
&+\left(\frac{1}{n} U^{\tau} U\right)^{-1} \frac{1}{T \sqrt{n}} \sum_{i=1}^{n} U_{i}^{\prime} v_{s}+o_{p}(1), \\
& \\
& \\
&
\end{aligned}
$$

where $\left\{\varepsilon_{i}\right\}$ is an unobservable sequence of independent identical distribution random variables with mean zero and variance one.

From $E\left(\nu_{s}\right)=0$ and the central limit theorem it follows that

$$
\frac{1}{T \sqrt{n}} \sum_{i=1}^{n} U_{i}^{\prime} \nu_{s} \stackrel{\mathrm{d}}{\longrightarrow} N\left(0, \sigma^{2} \Sigma\right) .
$$

On the other hand, the expectation of $1 /(T \sqrt{n}) \sum_{i=1}^{n} U_{i}^{\prime}$ $\sum_{j=i}^{n} \Delta t_{j}^{1 / 2} Z\left(t_{j}\right) \varepsilon_{j}$ does not converge to zero; that is to say, I have

$$
\begin{gathered}
\frac{1}{T \sqrt{n}} E\left(\begin{array}{c}
\sum_{i=1}^{n}\left[\left(\sum_{j=i}^{n} \Delta t_{j}\right)\left(\sum_{k=i-1}^{n} \Delta t_{k}^{1 / 2} Z\left(t_{k}\right) \varepsilon_{k}\right)\right] \\
\sum_{i=1}^{n}\left[\left(\sum_{j=i}^{n} \Delta t_{j} Y\left(t_{j}\right)\right)\left(\sum_{k=i-1}^{n} \Delta t_{k}^{1 / 2} Z\left(t_{k}\right) \varepsilon_{k}\right)\right] \\
\sum_{i=1}^{n}\left[\left(\sum_{j=i}^{n} \Delta t_{j} Z\left(t_{j}\right)\right)\left(\sum_{k=i-1}^{n} \Delta t_{k}^{1 / 2} Z\left(t_{k}\right) \varepsilon_{k}\right)\right]
\end{array}\right)=v, ~ \\
=\sqrt{\frac{T}{n^{4}}}\left(\begin{array}{c}
\sum_{i=1}^{n} \sum_{j=i-1}^{n} \sum_{k=1}^{n-j} E\left(Y\left(t_{j}\right) Z\left(t_{j+k}\right) \varepsilon_{j}\right) \\
\sum_{i=1}^{n} \sum_{j=i-1}^{n} \sum_{k=1}^{n-j} E\left(Z\left(t_{j}\right) Z\left(t_{j+k}\right) \varepsilon_{j}\right)
\end{array}\right)
\end{gathered}
$$

while $v \nrightarrow 0$. For simplicity, take the third competent as an example to estimate the value. From Lemma 1 of Politis and Romano [30], I can see that $Y_{t}, Z_{t}$ are both $\rho$-mixingdependent process and the mixing coefficient denoted by $\rho(l)$, too,

$$
\begin{aligned}
& \frac{1}{T \sqrt{n}} E \\
& \quad \times\left(\sum_{i=1}^{n}\left[\left(\sum_{j=i}^{n} \Delta t_{j} Z\left(t_{j}\right)\right)\left(\sum_{k=i-1}^{n} \Delta t_{k}^{1 / 2} Z\left(t_{k}\right) \varepsilon_{k}\right)\right]\right) \\
& =\sqrt{\frac{T}{n^{4}}} E\left(\sum_{i=1}^{n} \sum_{j=i-1}^{n} \sum_{k=1}^{n-j} Z\left(t_{j+k}\right) Z\left(t_{j}\right) \varepsilon_{j}\right) \\
& =\sqrt{\frac{T}{n^{4}}} \sum_{i=1}^{n} \sum_{j=i-1}^{n} \sum_{k=1}^{n-j} E\left(Z\left(t_{j}\right) Z\left(t_{j+k}\right) \varepsilon_{j}\right) \\
& =
\end{aligned}
$$

I can easily verify that, as $n \rightarrow \infty, 0<c_{1} \rho \leq(*) \leq c_{2}(\rho /(1-$ $\rho)$ ).

By condition (d), the variance is bounded uniformly for $i=1, \ldots, n$. Then

$$
\begin{aligned}
\left(\frac{1}{n} U^{\tau} U\right)^{-1} & \left\{\frac{1}{T \sqrt{n}} \sum_{i=1}^{n} U_{i}^{\prime} \sum_{j=i}^{n} \Delta t_{j}^{1 / 2} Z\left(t_{j}\right) \varepsilon\left(t_{j}\right)-v\right\} \\
\stackrel{\mathrm{d}}{\longrightarrow} & N\left(0, \Sigma^{-1} \Omega \Sigma^{-1}\right) .
\end{aligned}
$$


Summing up the above and the independency between $\varepsilon_{j}$ and $v_{s}$, I get

$$
\sqrt{n}\left(\widehat{\beta}-\beta-\sqrt{n}\left(U^{\tau} U\right)^{-1} v\right) \stackrel{\mathrm{d}}{\longrightarrow} N\left(0, \sigma^{2} \Sigma^{-1}+\Sigma^{-1} \Omega \Sigma^{-1}\right) .
$$

While the asymptotic bias $\sqrt{n}\left(U^{\tau} U\right)^{-1} v=o\left(\Sigma^{-1} v\right) \rightarrow 0$, therefore

$$
\sqrt{n}(\widehat{\beta}-\beta) \stackrel{\mathrm{d}}{\longrightarrow} N\left(0, \sigma^{2} \Sigma^{-1}+\Sigma^{-1} \Omega \Sigma^{-1}\right) .
$$

This completes the proof.

\section{Acknowledgments}

This paper was supported by NBRP (973 Program 2007CB814901) of China, NNSF Project (10771123) of China, RFDP (20070422034) of China, and NSF Projects (Y2006A13 and Q2007A05) of Shandong Province of China.

\section{References}

[1] J. M. Bismut, "Conjugate convex functions in optimal stochastic control," Journal of Mathematical Analysis and Applications, vol. 44, pp. 384-404, 1973.

[2] É. Pardoux and S. G. Peng, "Adapted solution of a backward stochastic differential equation," Systems \& Control Letters, vol. 14, no. 1, pp. 55-61, 1990.

[3] S. G. Peng, "Probabilistic interpretation for systems of quasilinear parabolic partial differential equations," Stochastics and Stochastics Reports, vol. 37, no. 1-2, pp. 61-74, 1991.

[4] É. Pardoux and S. Peng, "Backward stochastic differential equations and quasilinear parabolic partial differential equations," Stochastic Partial Differential Equations and Their Applications, Springer, vol. 176, pp. 200-217, 1992.

[5] É. Pardoux and S. Tang, "Forward-backward stochastic differential equations and quasilinear parabolic PDEs," Probability Theory and Related Fields, vol. 114, no. 2, pp. 123-150, 1999.

[6] S. Peng and Z. Wu, "Fully coupled forward-backward stochastic differential equations and applications to optimal control," SIAM Journal on Control and Optimization, vol. 37, no. 3, pp. 825-843, 1999.

[7] J. Ma and J. Yong, Forward-Backward Stochastic Differential Equations and Their Applications, vol. 1702 of Lecture Notes in Mathematics, Springer, Berlin, Germany, 1999.

[8] D. Nualart and W. Schoutens, "Backward stochastic differential equations and Feynman-Kac formula for Lévy processes, with applications in finance," Bernoulli, vol. 7, no. 5, pp. 761-776, 2001.

[9] D. Duffie and L. G. Epstein, "Stochastic differential utility," Econometrica, vol. 60, no. 2, pp. 353-394, 1992.

[10] N. El Karoui and M. C. Quenez, "Dynamic programming and pricing of contingent claims in an incomplete market," SIAM Journal on Control and Optimization, vol. 33, no. 1, pp. 29-66, 1995.

[11] L. Lin, F. Li, L. Zhu, and W. K. Härdle, "Mean volatility regressions," SFB 649 Economic Risk Berlin, Germany, 2010.

[12] M. C. Quenez, Méthodes de contrôle stochastique en finance [Thèse de doctorat], Université Pierre et Marie Curie, 1993.
[13] Ł. Delong, Backward Stochastic Differential Equations With Jumps and Their Actuarial and Financial Applications, EAA, Springer, New York, NY, USA, 2013.

[14] Y. Su and L. Lin, "Semi-parametric estimation for forwardbackward stochastic differential equations," Communications in Statistics, vol. 38, no. 11, pp. 1759-1775, 2009.

[15] X. Chen and L. Lin, "Nonparametric estimation for FBSDEs models with applications in finance," Communications in Statistics, vol. 39, no. 14, pp. 2492-2514, 2010.

[16] R. Stanton, "A nonparametric model of term structure dynamics and the market price of interest rate risk," The Journal of Finance, vol. 52, no. 5, pp. 1973-2002, 1997.

[17] B. Øksendal, Stochastic Differential Equations, Universitext, Springer, New York, NY, USA, 6th edition, 2003.

[18] M. Rosenblatt, "A central limit theorem and a strong mixing condition," Proceedings of the National Academy of Sciences of the United States of America, vol. 42, no. 1, pp. 43-47, 1956.

[19] M. Rosenblatt, "Density estimates and Markov sequencef," in Selected Works of Murray Rosenblatt, p. 240, Springer, New York, NY, USA, 2011.

[20] A. N. Kolmogorov and Y. A. Rozanov, "On strongmixing conditions for stationary gaussian processes," Theory of Probability \& Its Applications, vol. 5, no. 2, pp. 204-208, 1960.

[21] R. C. Bradley and W. Bryc, "Multilinear forms and measures of dependence between random variables," Journal of Multivariate Analysis, vol. 16, no. 3, pp. 335-367, 1985.

[22] Z. Lin and C. Lu, Limit Theory for Mixing Dependent Random Variables, vol. 378 of Mathematics and its Applications, Springer, New York, NY, USA, 1996.

[23] C. N. Morris, "Natural exponential families with quadratic variance functions," The Annals of Statistics, vol. 10, no. 1, pp. 65-80, 1982.

[24] G. M. Constantinides, "A theory of the nominal term structure of interest rates," The Review of Financial Studies, vol. 5, no. 4, pp. 531-552, 1992.

[25] J. Fan, "A selective overview of nonparametric methods in financial econometrics," Statistical Science, vol. 20, no. 4, pp. 317-357, 2005.

[26] J. Fan and C. A. Zhang, "A reexamination of diffusion estimators with applications to financial model validation," Journal of the American Statistical Association, vol. 98, no. 461, pp. 118-134, 2003.

[27] K. C. Chan, G. A. Karolyi, F. A. Longstaff, and A. B. Sanders, "An empirical comparison of alternative models of the short-term interest rate," The Journal of Finance, vol. 47, no. 3, pp. 1209-1227, 1992.

[28] Y. Aït-Sahalia, "Testing continuous-time models of the spot interest rate," The Review of Financial Studies, vol. 9, no. 2, pp. 385-426, 1996.

[29] N. El Karoui, S. Peng, and M. C. Quenez, "Backward stochastic differential equations in finance," Mathematical Finance, vol. 7, no. 1, pp. 1-71, 1997.

[30] D. N. Politis and J. P. Romano, "A general resampling scheme for triangular arrays of $\alpha$-mixing random variables with application to the problem of spectral density estimation," The Annals of Statistics, vol. 20, no. 4, pp. 1985-2007, 1992.

[31] X. Cui, W. Guo, L. Lin, and L. Zhu, "Covariate-adjusted nonlinear regression," The Annals of Statistics, vol. 37, no. 4, pp. 1839-1870, 2009. 
[32] M. Peligrad, "Properties of uniform consistency of the kernel estimators of density and of regression functions under dependence assumptions," Stochastics and Stochastics Reports, vol. 40, no. 3-4, pp. 147-168, 1992.

[33] T. Y. Kim and D. D. Cox, "Uniform strong consistency of kernel density estimators under dependence," Statistics \& Probability Letters, vol. 26, no. 2, pp. 179-185, 1996. 


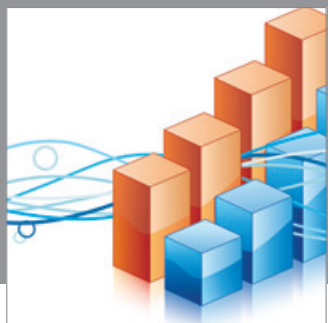

Advances in

Operations Research

mansans

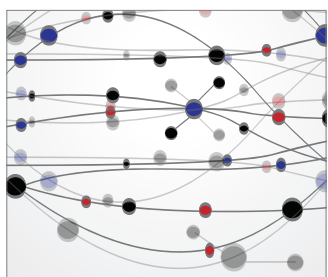

The Scientific World Journal
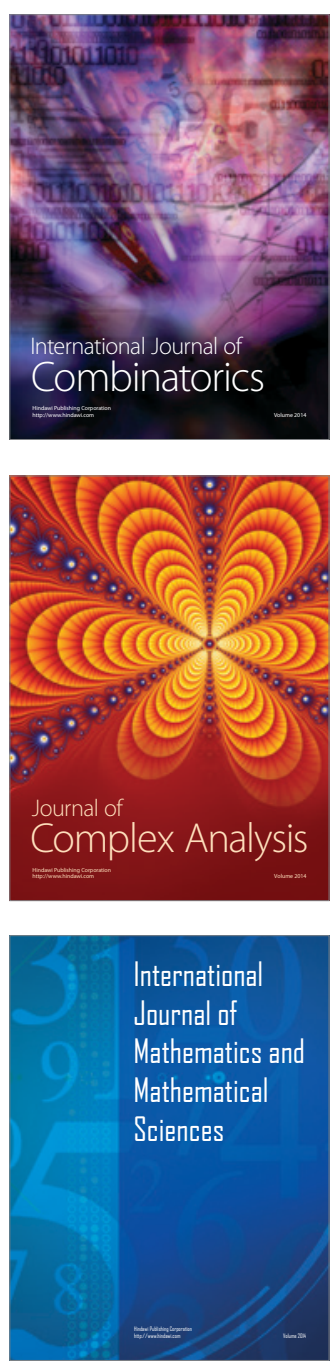
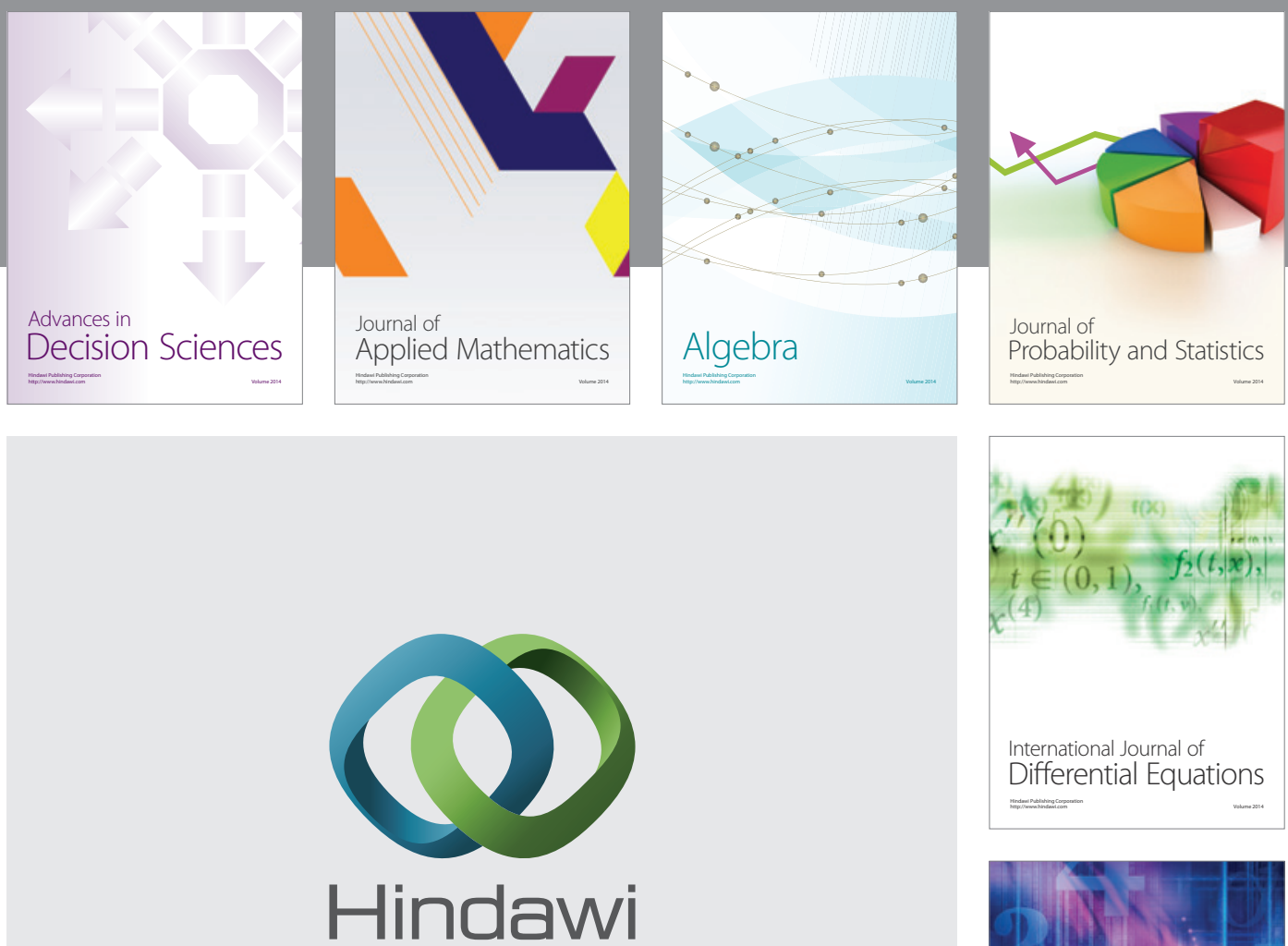

Submit your manuscripts at http://www.hindawi.com
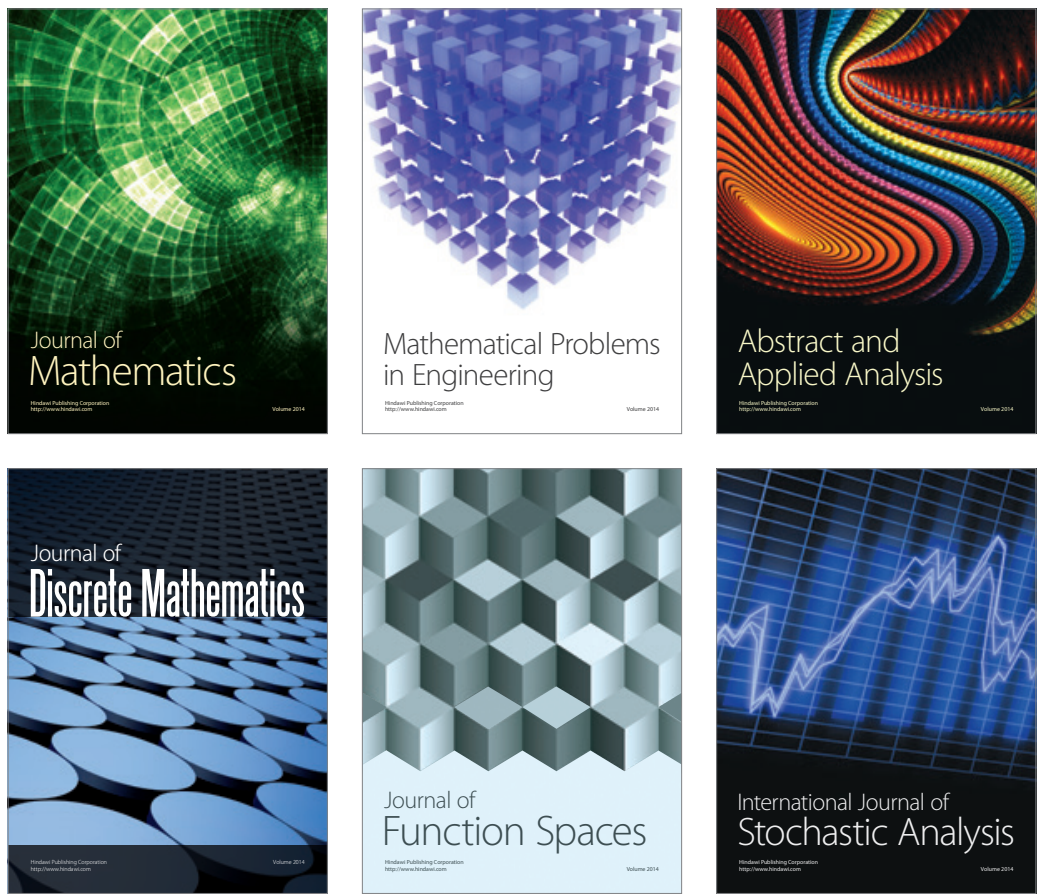

Journal of

Function Spaces

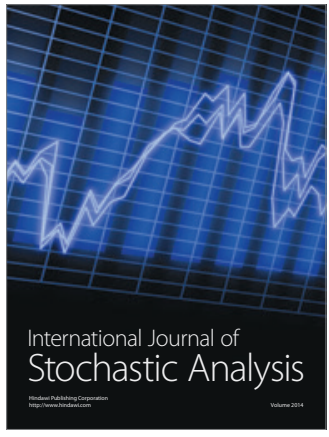

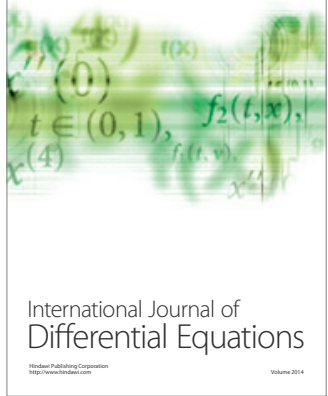
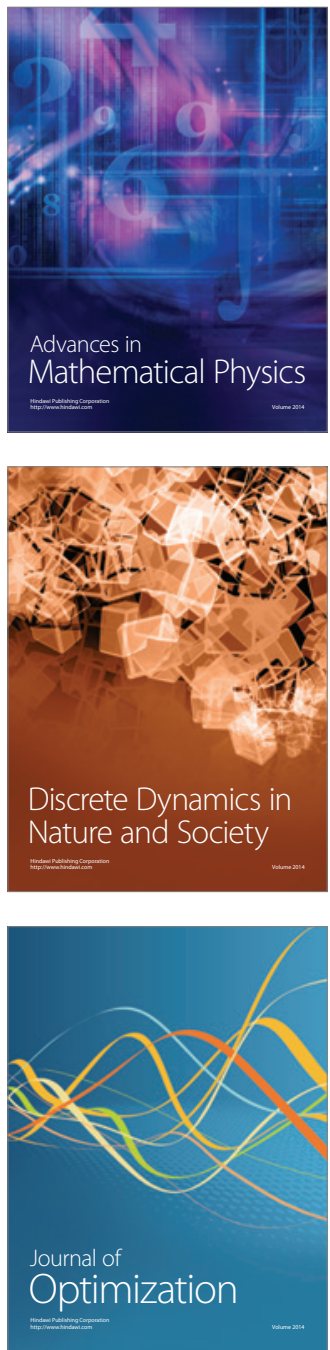